\title{
39 PRIORITY SETTING FOR END-OF-LIFE CARE
}

${ }^{1}$ Michael Spence, ${ }^{1}$ Rebecca Spencer, ${ }^{2}$ Janet Diffin, ${ }^{1}$ Peter Mellor, ${ }^{2}$ Gunn Grande. ${ }^{1}$ NIHR Collaboration for Leadership in Applied Health Research and Care (CLAHRC) Greater Manchester, UK; ${ }^{2}$ School of Nursing, Midwifery and Social Work, University of Manchester, UK

10.1136/bmjspcare-2016-001204.39

Introduction End-of-life (EoL) care is an under-researched area. It is therefore vital to focus on priorities that are likely to bring the most benefit to patients, carers, and families.

Aim This project aimed to identify EoL research priorities of Healthcare Professionals (HCPs) and carers in Greater Manchester.

Methods Following a scoping exercise of EOL services in Greater Manchester, CLAHRC GM identified six EOL local research topics for further exploration within the 10 national priorities outlined by PeolcPSP.

- Education and knowledge for staff and carers

- Access to 24-hour care and support

- Equitable Access

- Advance care planning

- Care at home

- Continuity of care

CLAHRC GM held a priority setting event to consult with 32 HCPs and separate consultations with 26 carers facilitated by local carer groups from Manchester, Salford, and Bury. Carers and HCPs selected their priorities from the topics.

Results Combining carer and HCP responses, the topics of shared priority were:

- Advance care planning

- Access to 24-hour care

- Education and knowledge for staff and carers

Whilst carers emphasised training and education as the priority and HCPs focused more on advance care planning, it was clear that all topics had importance to both groups.

HCPs also identified important research questions within their chosen topics.

Conclusions This study provides successful procedures for engaging with stakeholders to develop local priorities to guide EoL research of relevance to HCPs and user representatives.

\section{REFERENCE}

1 Brereton L, Ingelton C, Gardiner C, et al. Lay and professional stakeholder involvement in scoping palliative care issues: methods used in seven European countries. Palliat Med 2016 